\title{
String breaking by dynamical fermions in three-dimensional lattice QCD
}

\author{
Howard D. Trottier \\ Newman Laboratory of Nuclear Studies, Cornell University, Ithaca, NY 14853-5001, and \\ Physics Department, Simon Fraser University, Burnaby, B.C., Canada V5A 1 S6⿴囗十
}

\begin{abstract}
The first observation is made of hadronic string breaking due to dynamical fermions in zero temperature lattice QCD. The simulations are done for $\mathrm{SU}(2)$ color in three dimensions, with two flavors of staggered fermions. The results have clear implications for the large scale simulations that are being done to search (so far, without success) for string breaking in four-dimensional QCD. In particular, string breaking is readily observed using only Wilson loops to excite a static quark-antiquark pair. Improved actions on coarse lattices are used, providing an extremely efficient means to access the quark separations and propagation times at which string breaking occurs.
\end{abstract}


The string picture of quark confinement predates Quantum Chromodynamics, and continues to play a central role in theoretical efforts to characterize the physics of QCD [1]. Simulations of quenched lattice QCD have in fact demonstrated that color-electric field lines connecting a static quark and antiquark are squeezed into a narrow tube [2], in accord with many models of the linearly rising static potential.

In QCD with dynamical fermions the flux-tube joining static quarks is expected to be unstable against fission at large $R$, where there is enough energy in the fields to materialize light quarks from the vacuum, which bind to the heavy quarks to form a pair of colorneutral bound states. The simplest indicator of string breaking is provided by the static quark-antiquark potential $V(R)$, which should approach a constant at large $R$

$$
V(R \rightarrow \infty)=2 M_{Q \bar{q}}
$$

where $M_{Q \bar{q}}$ is the mass of a bound state of a heavy quark and a light antiquark.

There is as yet no convincing evidence of string breaking in lattice simulations at zero temperature, despite extensive simulations by several large scale collaborations [1,3] 6]. A recent conjecture [7,8], which has received widespread attention, holds that the failure to detect the broken string state is due to a very poor overlap of the Wilson loop operator with the true ground state of the system at large $R$ (Wilson loops have been used almost exclusively to construct trial states in these simulations). The possibility has been raised that the broken string state may even be unobservable with Wilson loops for all practical purposes [3]. This picture has recently stimulated interest in simulations using other operators to excite the system [9, 10].

Evidence for a somewhat different point of view is presented in this paper. The task of observing string breaking in full QCD is computationally very challenging, given the high cost of simulating dynamical fermions. These simulations have so far been done on lattices with a relatively fine grid. Consequently, only a fairly limited range of quark separations $R$ has been explored and, more importantly, the propagation of the trial states created by Wilson loops has only been studied for rather short Euclidean times $T$ (e.g., $T=0.4-0.8 \mathrm{fm}$ in Ref. [3]). The restriction to such short propagation times precludes a definitive assessment of whether string breaking occurs in these states. This limitation may be substantially alleviated by working on coarser lattices with improved actions.

This proposal is assessed here by doing simulations in three-dimensional lattice QCD $\left(\mathrm{QCD}_{3}\right)$ with two flavors of dynamical staggered fermions. $\mathrm{QCD}_{3}$ provides an excellent laboratory for studying the physics of realistic QCD (for a review see Ref. [11]). Quenched $\mathrm{QCD}_{3}$ has been shown to exhibit linear confinement, flux-tube formation, a deconfining phase transition, and a rich glueball spectrum, and spontaneous breakdown of a "chiral" symmetry has been observed in both the quenched and unquenched theories [12].

The first observation of string breaking by dynamical fermions is reported here, using only Wilson loop operators to excite the static quark-antiquark system [13]. Although these simulations are done in $\mathrm{QCD}_{3}$, the results have clear implications for work on the four-dimensional theory. In particular the simulations are done on coarse lattices, using an improved gluon action that is accurate through $O\left(a^{2}\right)$, and a staggered fermion action that is accurate through $O(a)$. This allows the system to be much more efficiently probed at 
the physical separations and propagation times relevant to string breaking. Moreover, the overlap of an operator with the lowest-lying state generally increases with the coarseness of the lattice, due to the suppression of higher momentum modes in the trial state. In contrast, recent studies of $\mathrm{QCD}_{3}$ with dynamical scalar matter fields [9], and adjoint matter fields [14, using unimproved actions on lattices with about half the spacing considered here, did not resolve string breaking in Wilson loops, presumably because insufficient propagation times were attained.

Using the quenched string tension to set the "physical" length scale for $\mathrm{QCD}_{3}$, these results suggest that string breaking occurs in Wilson loops at modest propagation times, somewhat in excess of $1 \mathrm{fm}$ (on modestly coarse lattices), which is not much beyond what has been reached in four dimensions. The physical quark separation $R \approx 1.5 \mathrm{fm}$ at which string breaking is found in $\mathrm{QCD}_{3}$ is consistent with expectations in four dimensions [15. Lattice spacings of about $0.2-0.3 \mathrm{fm}$ are used here; these lattices are about twice as coarse as those that have been used in four-dimensional studies of string breaking. Comparable lattice spacings could be reliably accessed in realistic QCD using improved actions [16], resulting in an enormous improvement in computational efficiency.

To begin with, consider the pure gauge theory in three dimensions. To reduce the computational cost $\mathrm{SU}(2)$ color is considered. Simulations were done using a tree-level $O\left(a^{2}\right)$-accurate gluon action, allowing for different spatial and temporal lattices spacings, $a_{s}$ and $a_{t}$ respectively 17

$$
\mathcal{S}_{\text {imp }}=-\beta \sum_{x, \mu>\nu} \xi_{\mu \nu}\left[\frac{5}{3} P_{\mu \nu}-\frac{1}{12}\left(R_{\mu \nu}+R_{\nu \mu}\right)\right] .
$$

$P_{\mu \nu}$ is one-half the trace of the $1 \times 1$ plaquette and $R_{\mu \nu}$ is one-half the trace of the $2 \times 1$ rectangle in the $\mu \times \nu$ plane. The bare QCD coupling $g_{0}^{2}$ (of dimension 1 ) enters through the dimensionless parameter $\beta=4 / g_{0}^{2} a_{s}$, and the bare lattice anisotropy is input through $\xi_{3 i}=\xi_{i 3}=a_{s} / a_{t}$ and $\xi_{i j}=a_{t} / a_{s}(i, j=1,2)$.

$\mathrm{QCD}_{3}$ is a super-renormalizable theory; in fact the bare coupling $g_{0}^{2}$ and bare quark masses $m_{0}$ remain finite as the ultraviolet regulator is removed. Since $g_{0}^{2}$ and $m_{0}$ are cutoffindependent in the continuum limit, one obtains extremely simple scaling laws for physical quantities in lattice $\mathrm{QCD}_{3}$. In particular, masses in lattice units (including the input bare quark masses) should satisfy

$$
\text { Bam }=\text { constant }
$$

in the continuum limit. The improved action $S_{\text {imp }}$ differs from the continuum theory at the tree-level by terms of $O\left(a^{4}\right)$; one-loop corrections induce errors of $O\left(g_{0}^{2} a^{3}\right)$.

Results for the quenched string tension obtained with $S_{\text {imp }}$ are shown in Fig. 1; for comparison, results obtained by Teper [1] for the unimproved Wilson gluon action are also shown (the dashed lines show the continuum limit estimated in [11]). A comparison of the potential computed from the two actions on lattices of comparable spacing is shown in Fig. 2.

Simulations of $S_{\text {imp }}$ were done on lattices with $\beta=2,2.5$, and 3 , all with anisotropy $a_{t} / a_{s}=1 / 4$. The lattice volumes range from $16^{3}$ at $\beta=2$ to $24^{3}$ at $\beta=3$. Hybrid molecular dynamics were used to generate the configurations (the $\Phi$ algorithm 18 was used to generate the unquenched configurations analyzed below); 50 molecular dynamics steps 
were taken with step size $\Delta t=0.02$. A standard fuzzing procedure was employed on the link variables used in Wilson loop measurements. Integrated autocorrelation times satisfied $\tau_{\text {int }} \lesssim 0.5$, with typically $5-10$ trajectories skipped between measurements.

A superficially surprising aspect of these results is the $O(a)$ scaling violation evident in the Wilson action data for $\sqrt{\sigma} / g_{0}^{2}$. In fact this demonstrates the need to renormalize the bare coupling at finite $a$ [19]. In four dimensions it is known that perturbative expansions in the bare lattice coupling are spoiled by large renormalizations. A renormalized coupling defined by a physical quantity absorbs these large corrections, and greatly improves perturbation theory for many quantities [20].

In lattice $\mathrm{QCD}_{3}$ one expects to find an $O\left(g_{0}^{2} a\right)$ renormalization of the bare coupling at one-loop order. This produces the linear scaling violation in the unimproved results for $\sqrt{\sigma} / g_{0}^{2}$. On the other hand this effect can be removed by using a physical quantity to define a renormalized scale. For example, Teper has done simulations of three-dimensional glueball masses $m_{G}$ [1], and showed that the ratios $m_{G} / \sqrt{\sigma}$ exhibit $O\left(a^{2}\right)$ scaling violations.

The distinctive signature of the renormalization of the bare coupling in $\mathrm{QCD}_{3}$ exposes an interesting feature of the action. One might expect to see a reduction in the renormalization of $g_{0}^{2}$ when $S_{\text {imp }}$ is used, compared to simulations with the Wilson action. Remarkably one finds that the renormalization is in fact almost completely eliminated; as seen in Fig. 11 the error in $\sqrt{\sigma} / g_{0}^{2}$ at $\beta=2$ is reduced from about $45 \%$ with the Wilson action, to less than about $5 \%$ with $S_{\mathrm{imp}}$. This is also apparent from the data in Fig. 2, where the potential $V$ and separation $R$ from the lattices at $\beta=2$ and 3 are compared on a common scale, set by $g_{0}^{2}$. This is a genuinely surprising result, since all of the higher dimension operators present in $S_{\text {imp }}$ should contribute to a leading $O\left(g_{0}^{2} a\right)$ renormalization of the bare coupling. Apparently the operator series in this effective action converges very rapidly, even at scales near the lattice cutoff.

One can therefore conveniently measure physical quantities directly in terms of the bare coupling when the improved action Eq. (2) is used. It is important to note however that the use of $S_{\text {imp }}$ would prove advantageous even if $g_{0}^{2}$ required renormalization; one would simply use a measured quantity (such as the quenched string tension) to set the scale for other observables, and the leading $O\left(a^{2}\right)$ errors in the Wilson action would thus be removed.

Simulations with dynamical Kogut-Susskind fermions were done to look for effects of string breaking. The staggered fermion action $\mathcal{S}_{\mathrm{KS}}$ in three dimensions [21] is identical in form to the four-dimensional theory, and describes two flavors of four-component spinors

$$
\begin{gathered}
\mathcal{S}_{\mathrm{KS}}=\sum_{x, \mu} \eta_{\mu}(x) \bar{\chi}(x)\left[U_{\mu}(x) \chi(x+\hat{\mu})\right. \\
\left.-U_{\mu}^{\dagger}(x-\hat{\mu}) \chi(x-\hat{\mu})\right]+2 a m_{0} \sum_{x} \bar{\chi}(x) \chi(x),
\end{gathered}
$$

where $\eta_{\mu}(x)=(-1)^{x_{1}+\ldots+x_{\mu-1}}$ is the usual staggered fermion phase. Unquenched simulations were done with improved glue on isotropic lattices $\left(a_{t}=a_{s}\right)$ at $\beta=2$ and 3 , on $12^{2} \times 8$ and $16^{2} \times 10$ volumes respectively [22]. Isotropic lattices for the unquenched simulations were used in order to probe the longest propagation times $T$ possible, for the least computational cost (although measurements over a finer range of $T$ on anisotropic lattices would be advatangeous for fitting and possible extraction of excited state energies). The input bare quark mass in lattice units was scaled according to $m_{0} / g_{0}^{2}=0.075$ [cf. Eq. (3)], with $m_{\pi} / m_{\rho}$ 
found to be $\approx 0.75$. Approximately 6,000 measurements were done at $\beta=2$, and 2,000 at $\beta=3$.

The leading discretization errors in the action Eq. (41) are of $O\left(a^{2}\right)$ [23]; at finite lattice spacing the bare quark mass must also absorb an $O\left(g_{0}^{2} a\right)$ renormalization. However these effects appear to induce little scaling violation in the static potential at the small quark mass used here, perhaps because the energy in the color fields dominates the string breaking process (significant scaling violations in the unquenched potential were seen when the Wilson gluon action was used).

Results for the unquenched potential are shown in Fig. 3. The onset of string breaking is assessed by comparing the potential energy with the mass of the two $Q \bar{q}$ bound states into which the system should hadronize. The staggered heavy-light meson propagator [24] was computed in the unquenched configurations at $\beta=3$, for equal valence and sea quark masses; the result is shown as the dashed lines in Fig. 3. String breaking is clearly demonstrated, with the unquenched potential approaching the expected asymptotic value [cf. Eq. (11)]. Excellent scaling behavior is observed; note that the lattice spacing increases by $50 \%$ from $\beta=3$ to $\beta=2$. A continuum extrapolation of the string breaking distance $R_{b}$ can be estimated from these results, $R_{b} /(\beta a) \approx 2.5$.

The onset of string breaking is made particularly evident by a comparison of the quenched and unquenched potentials at the same $\beta$ (note that no adjustment of the energy zero has been made in any of these results). The meaningfulness of this comparison is supported by the fact that the bare coupling $g_{0}^{2}$ undergoes little renormalization; moreover quenching effects at a given $\beta$ were found to change the $\rho$-meson mass by less than $10 \%$.

Only (fuzzy) Wilson loop operators were used in these calculations. As discussed above, little evidence of string breaking in Wilson loops has been found in previous simulations with dynamical fermions. A distinguishing feature of the simulations done here, compared with earlier work, is the relative coarseness of the lattices that were used (of course the physics of the string breaking process could be substantially different in three dimensions; on the other hand, simulations of $\mathrm{QCD}_{3}$ on much finer lattices [9.14] did not resolve string breaking in Wilson loops).

Using coarse lattices made it much easier to measure Wilson loops $W(R, T)$ at propagation times $T$ large enough to accurately identify the ground state energy at large $R$. Plots of the time-dependent effective potential $V(R, T)=-\ln [W(R, T) / W(R, T-1)]$ are given

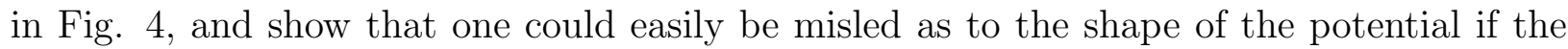
calculation is not done at large enough $T$, particularly at large $R$.

In order to have some intuition for the length scales probed in these simulations, it is instructive to use the physical value of the string tension in four dimensions, $\sqrt{\sigma}=0.44 \mathrm{GeV}$, to in effect set the value of the dimensionful coupling constant in $\mathrm{QCD}_{3}$. Using the quenched continuum extrapolation $\beta a \sqrt{\sigma}=1.33(1)$ [11] one identifies, for example, $a(\beta=3) \approx 0.2 \mathrm{fm}$. Comparable, if slightly smaller, estimates of the lattice spacing are obtained if simulation results for the quenched or unquenched $\rho$ meson mass in $\mathrm{QCD}_{3}$ are identified with the physical mass.

This scale setting implies a string breaking distance $R_{b} \approx 1.5 \mathrm{fm}$, which is numerically very similar to estimates of $R_{b}$ in four dimensions [15]. The results of Fig. [1 suggest that propagation times somewhat in excess of $1 \mathrm{fm}$ are needed to adequately resolve string breaking in Wilson loops on modestly coarse lattices; notice than an appreciable rise in the 
potential remains even at $T \approx 0.6 \mathrm{fm}$, which is comparable to the longest propagation times resolved in the four-dimensional simulations of Ref. [3].

Although longer propagation times $T$ are necessary to fully isolate the ground state energy at the largest separations $R$ in Fig. 4 , these data nevertheless clearly support the onset of string breaking. This follows from a comparison of the unquenched and quenched potentials. Of particular importance is the fact that any slope that may exist in the unquenched potential data at large $R$ is clearly much smaller than the slope in the quenched data. Such a small slope, if truly present, would imply a very large change in other physical quantities due to unquenching. In fact, $m_{\rho}$ was found to change by less than $10 \%$ with unquenching. Hence the significant flattening of the unquenched potential data in Figs. 3 and 4 , compared to the quenched data, is very strongly indicative of the onset of string breaking.

To summarize, string breaking due to dynamical fermions was observed in threedimensional lattice QCD. The results have clear implications for current work on the four dimensional theory. In particular, string breaking was readily observed using only Wilson loop operators. The use of improved actions on coarse lattices provided a crucial advantage in accessing the quark separations and propagation times at which string breaking occurs. The use of comparably coarse lattices in four dimensions would result in an enormous improvement in computational efficiency, and could enable string breaking to be demonstrated in realistic $\mathrm{QCD}$.

I am indebted to Peter Lepage for several insightful discussions. I also thank $\mathrm{R}$. Woloshyn, R. Fiebig, M. Alford, and M. Teper for fruitful conversations. This work was supported in part by the Natural Sciences and Engineering Research Council of Canada. 


\section{REFERENCES}

* Permanent address.

[1] For a recent review see, e.g., J. Kuti, Report No. hep-lat/9811021.

[2] See C. Michael, Report No. hep-ph/9710249, and references therein.

[3] S. Aoki et al., CP-PACS collaboration, Report No. hep-lat/9809185.

[4] M. Talevi et al., UKQCD collaboration, Report No. hep-lat/9809182.

[5] G. Bali et al., SESAM and T $\chi$ L collaborations, Nucl. Phys. B (Proc. Suppl.) 63, 209 (1998).

[6] String breaking due to dynamical fermions at nonzero temperature has been recently been reported in C. DeTar et al., Report No. hep-lat/9808028.

[7] S. Güsken, Nucl. Phys. B (Proc. Suppl.) 63, 16 (1998).

[8] I. T. Drummond, Phys. Lett. B 434, 92 (1998).

[9] O. Philipsen and H. Wittig, Phys. Rev. Lett. 81, 4056 (1998).

[10] F. Knechtli and R. Sommer, Phys. Lett. B 440, 345 (1998); C. Stewart and R. Koniuk, hep-lat/9811012.

[11] M. Teper, Phys. Rev. D 59, 014512 (1999).

[12] E. Dagotto, A. Kocic and J.B. Kogut, Nucl. Phys. B 362, 498 (1991).

[13] Some this work was reported in preliminary form in H. D. Trottier, Report No. heplat/9809183.

[14] G. I. Poulis and H. D. Trottier, Phys. Lett. B 400, 358 (1997).

[15] C. Alexandrou et al., Nucl. Phys. B 414, 815 (1994).

[16] See, e.g., M. Alford, T. R. Klassen, and G. P. Lepage, Phys. Rev. D 58, 034503 (1998).

[17] K. Symanzik, Nucl. Phys. B 226, 187 (1983); M. Lüscher and P. Weisz, Comm. Math. Phys. 97, 59 (1985).

[18] S. Gottlieb et al., Phys. Rev. D 35, 2531 (1987).

[19] This argument is due to G. P. Lepage (private communication). See also G. D. Moore, Nucl. Phys. B 523, 569 (1998).

[20] G.P. Lepage and P.B. Mackenzie, Phys. Rev. D 48, 2250 (1993).

[21] C. Burden and A.N. Burkitt, Europhys. Lett. 3, 545 (1987).

[22] Finite temperature effects should be negligible on these lattices, where $1 /\left(N_{\tau} a\right) \lesssim 0.2 T_{c}$ [using the quenched estimate of $T_{c}$ in M. Teper, Phys. Lett. B 313, 417 (1993)].

[23] Simulations were also done with some improvement of the staggered fermion action; see Ref. 13.

[24] A. Mihaly et al., Phys. Rev. D 55, 3077 (1997). 


\section{FIGURES}

FIG. 1. Lattice spacing dependence of the quenched string tension from the improved gluon action $(\square)$; results for the Wilson gluon action $(\bullet)$ are taken from Ref. 11].

FIG. 2. Static potential from the Wilson gluon action at $\beta=2(\bullet)$, and from the improved gluon action at $\beta=2(\square)$ and $\beta=3(\times)$.

FIG. 3. Static potential from the improved gluon action with and without dynamical staggered fermions, at $\beta=2(\square)$ and $\beta=3(\times)$. The unquenched simulations were done on isotropic lattices $\left(a_{t}=a_{s}\right)$. The dashed lines are one sigma limits for $2 \beta a_{s} M_{Q \bar{q}}$, computed on dynamical configurations at $\beta=3$.

FIG. 4. Time-dependent effective potential $V(R, T)$ versus $R$, in the dynamical configurations at $\beta=3$, for $T / a_{s}=1(\square), 3(\circ)$, and $5(\triangle)$. Also shown is the quenched potential at $\beta=3(\bullet)$, and the broken string energy (dashed lines). 


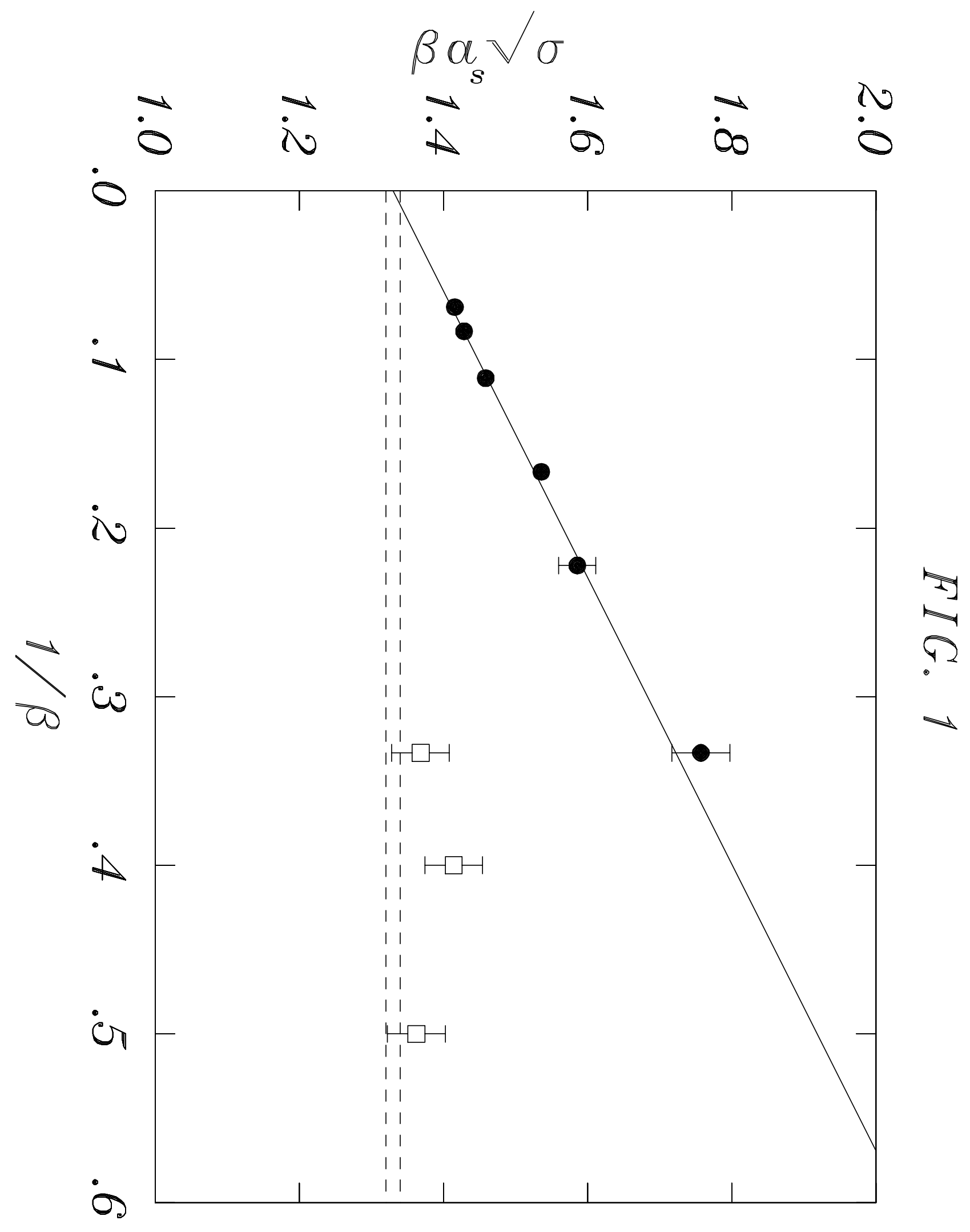




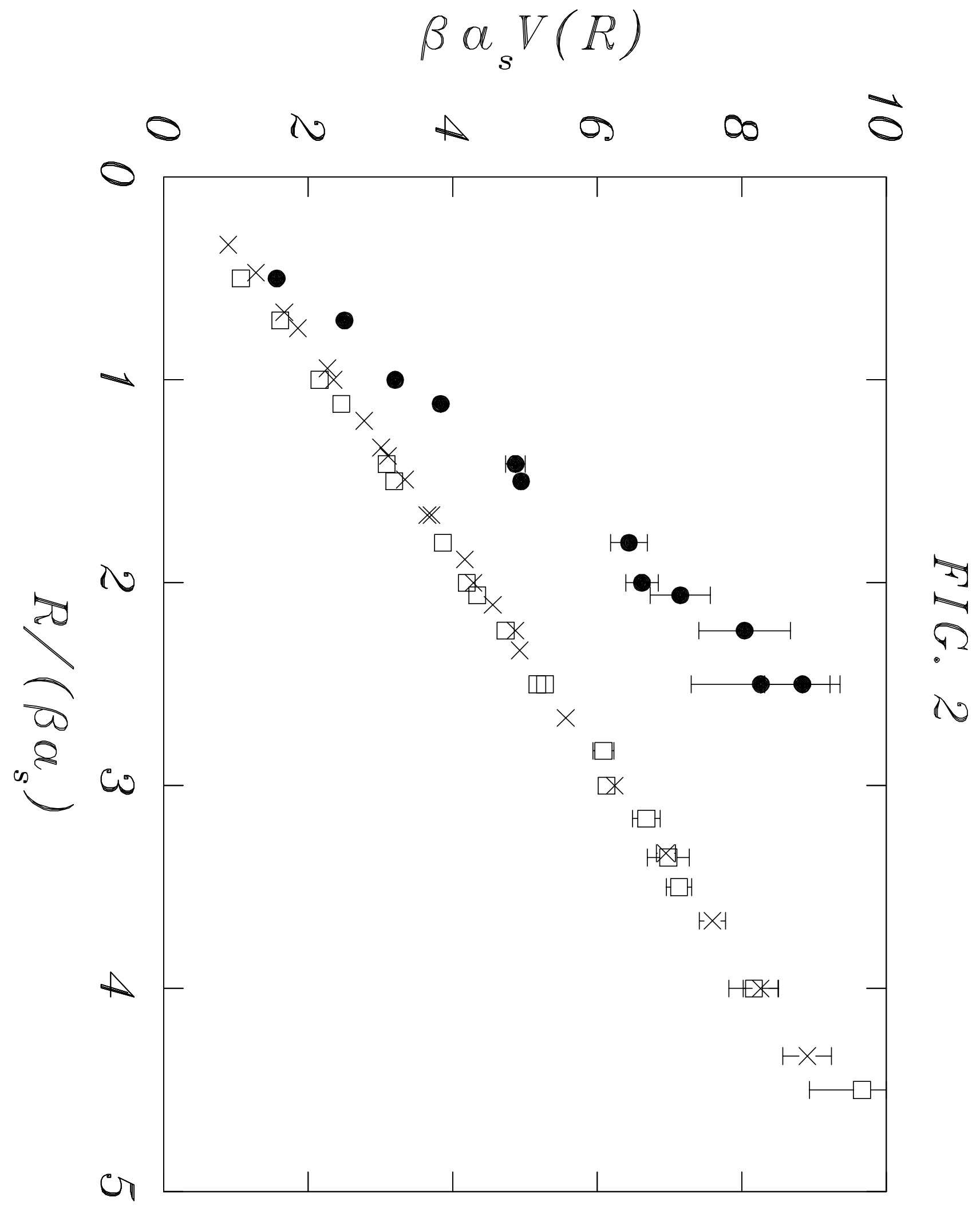




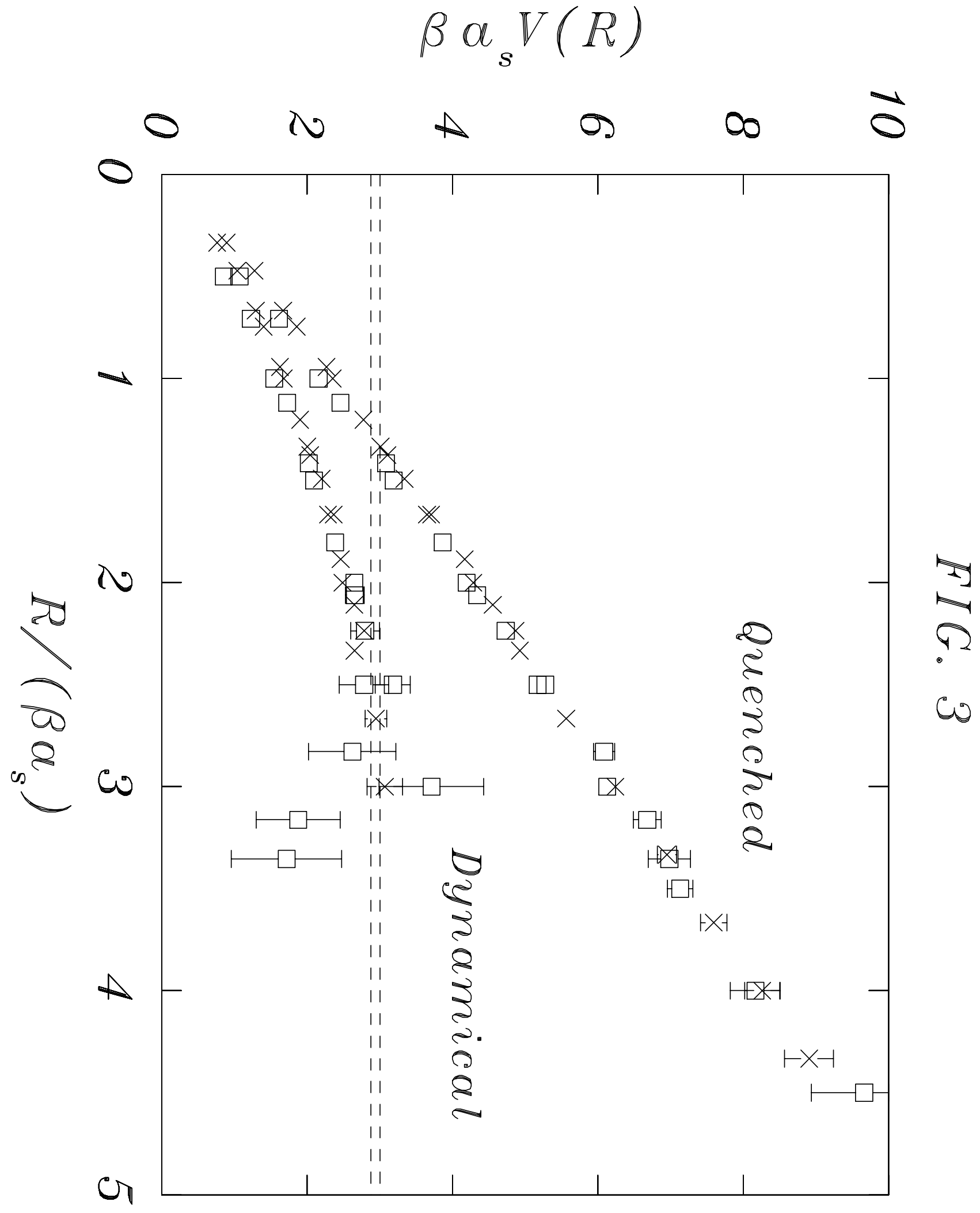




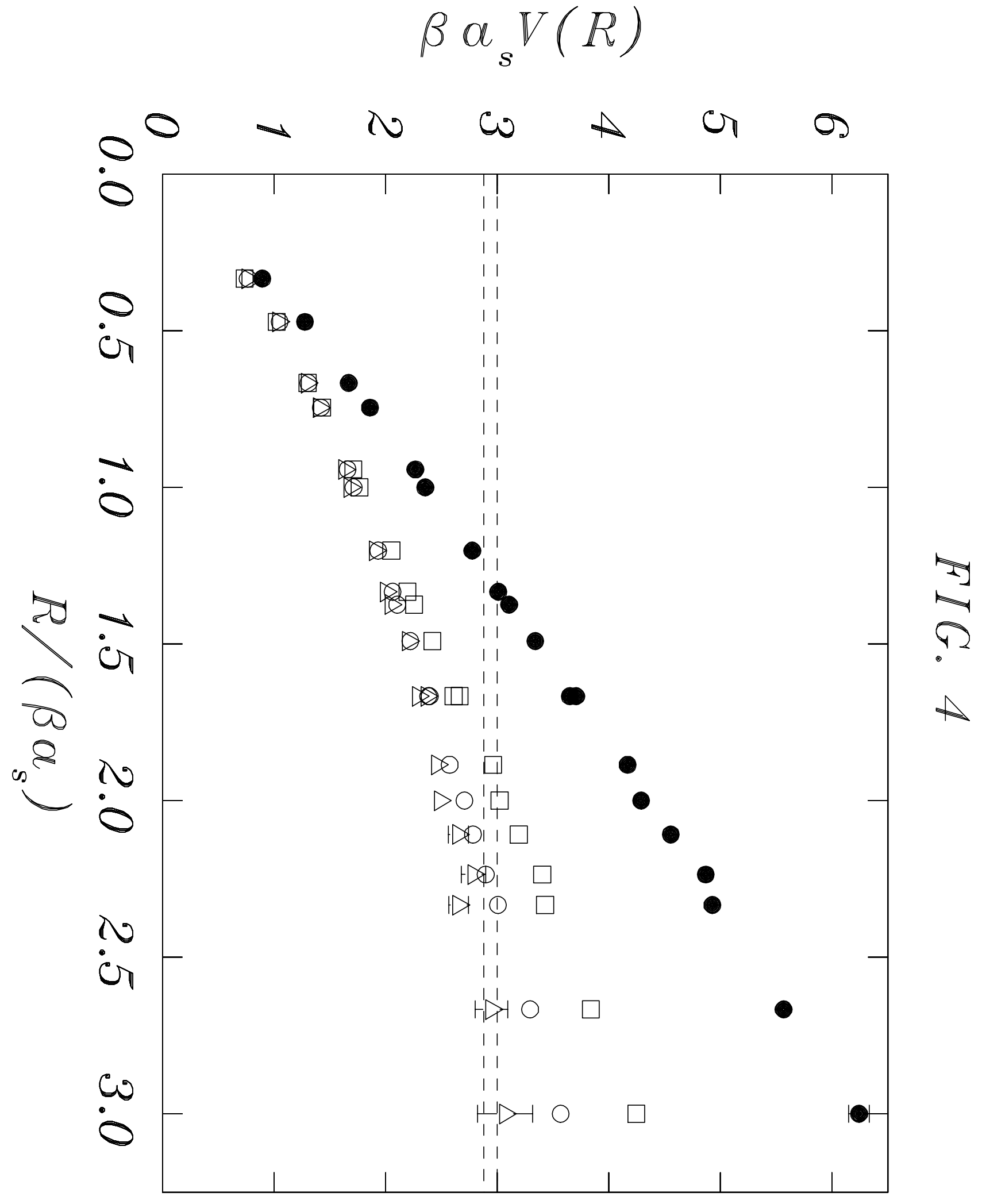

